\title{
A Computer-Aided Technique for Planning Plastic Surgery Based on 3D Face Scans: Preliminary Results.
}

\author{
Andrea BOTTINO, Matteo DE SIMONE*, Aldo LAURENTINI \\ Dipartimento Automatica e Informatica, Politecnico di Torino, Turin, Italy
}

\begin{abstract}
Plastic surgery plays a major role in today's health care. Planning plastic facial surgery requires dealing with the elusive concept of attractiveness for evaluating aesthetically effective outcomes for a particular face. A number of computer tools have been proposed for previewing possible surgery results and allowing their aesthetical evaluation by surgeons and patients which are based on the manual warping of $2 \mathrm{D}$ images or $3 \mathrm{D}$ scans. How to manipulate faces, as well as the evaluation of the results, are left to the surgeon's judgment. In a previous work, we proposed a quantitative approach able to automatically suggest effective patient-specific improvements of facial attractiveness and we applied it to 2D face profiles. In this paper we describe the application of the approach to 3D scans, and present some preliminary results.
\end{abstract}

Keywords: planning plastic surgery, 3D face scan, automatic beautification

\section{Introduction}

Planning reconstructive and cosmetic surgery requires taking into account a number of physiological and psychological constraints: age, sex, health state, structure and shape of the face but also patient's needs and expectations. The frequency of secondary rhinoplasty, that is nose operations carried out to correct or revise an unsatisfactory outcome from a previous rhinoplasty, which is estimated around $8 \%-15 \%$, shows that correct planning is far from simple [1].

A number of computer-assisted solutions have been proposed for previewing surgery results and allowing their aesthetical evaluation by surgeons and patients. Most of these support the user in depicting possible outcomes of the surgery. They rely on 2D front and profile images ([2,3]) or on 3D scans of the patient's face $([4,5,6])$ and essentially provide morphing tools for 2D images or 3D scans elaboration. Some of these systems take into account to some extent surgical constraints [2]. Anyway, how to effectively change face shape, as well as the evaluation of the results, are left to the surgeon's judgment. Instead, our efforts are addressed to implement a computer system able to support the human judgment by suggesting how to enhance attractiveness. For this purpose we do not need a quantitative approach to assess beauty as much as we need a quantitative approach to improve beauty.

In recent years the scientific analysis of facial attractiveness has been a major issue for researchers in fields such as psychology, psychobiology, anthropology, evolutionary biology, behavioral and cognitive sciences, and in medical areas such as plastic surgery and orthodontics. Many thousands of papers on the subject have been presented in these areas. One empirical result must be highlighted: beauty is not, or not only, in the eye of the beholder. Empirical rating studies have demonstrated high beauty rating congruence over ethnicity, social class, age, and sex (see for instance $[7,8,9,10])$. This means that the human perception of facial attractiveness is essentially data-driven, and largely irrespective of the perceiver. These findings suggested that Computer Vision and Image Processing techniques could be used for the objective analysis of facial attractiveness.

Several recent researches aimed at analyzing beauty and finding the measurable elements more representative of facial attractiveness have been reported. For assessing beauty, as for identity recognition [11], two approaches are mainly used: the feature approach and the appearance based or holistic approach. The former, in a simplified form, was used for centuries by sculptors, painters and, as a rough working guide, by plastic surgeons $[12,13]$. It stems from the idea of ideal proportions, or beauty canon, for the human body, dating back to the Greek sculptor Polycletus. Recent computer vision and image processing researches construct a feature vector using the distances between face landmarks, angles between lines joining the landmarks, and other parameters such as texture in selected areas $[12,14,15,16,17,18]$. Such vectors, a point in a multidimensional face space, have been compared with classic beauty canons, or, more effectively, with samples of beautiful faces. The holistic approach reduces the dimensionality of the image pixel vector with techniques as PCA [16,19]. 2D face images beautification for photo retouching is also reported [20]. 
The literature shows that automatically rating attractiveness with results similar to those provided by human observers is a complex matter. In particular, the problem of establishing which facial features are more relevant to attractiveness is far from solved.

In a previous paper we presented a general approach to deal with the elusive concept of attractiveness and to support the human judgment by automatically suggesting shape changes effective in improving attractiveness of a particular subject. The approach has been applied to 2D images. As far as the authors know, up to now no other similar research for supporting plastic surgery has been presented. In this paper we present a further development, concerning a planning system based on 3D face scans. The proposed approach requires the availability of a database of 3D scans of attractive faces. Since such a database is not currently available nor in a public accessible form nor as a commercial product, we will also describe our work on collecting high resolution samples for building a DB of attractive faces that will be soon made available to the scientific community.

The paper is organized as follows. In Section 2 we describe the general idea for face beautification and its rationale. In Section 3 we describe how this approach can be applied to 3D scans, and the current state of the project.

\section{Our approach to beautification}

We underlined that, in spite of much research in the area, the problem of understanding which facial elements are more effective for assessing facial beauty is far from solved. However, our goal is approaching harmonious shapes, which fortunately is easier.

Observe first that several empirical results support the idea that there is not a unique beauty prototype [18]. Although face images obtained by averaging several facial images are usually rated more attractive than each component image, attractive faces are not average [21,22]. Beauty ratings have been found largely independent on ethnicity, but faces rated beautiful can be rather different, as well as their proportions, in Caucasian, Chinese or African groups [7,23], or even within these groups. Cognitive theories support the idea that humans define and use several category prototypes for face image analysis [24].

On the basis of these results, our general idea for patient-specific beautification is to consider many attractive, or at least regular, face prototypes, and to suggest surgical procedures able to approach the prototypes closer to the patient's face. Observe that the idea of many samples of attractive, or harmonic face also implies that in general there is not a unique prototype of a particular facial feature (e. g. mouth, nose), but there could be different shapes that are more or less attractive, depending on the perceived general harmony and integration with the rest of the face [25] (Fig. 1).
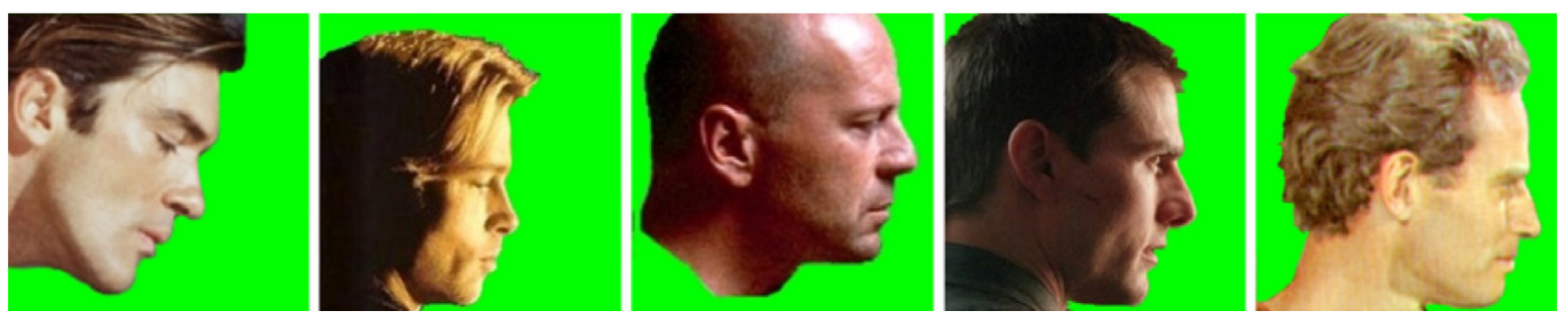

Fig.1. Different shapes could be more or less attractive, depending on the perceived general harmony

In more detail, our approach to the face beautification works as follows:

1. consider a particular (unpleasant or disharmonic) patient's face feature (target feature), and compare the face of the patient with many faces rated beautiful (reference database), excluding that particular feature;

2. minimize some convenient distance measure in the face space and find the nearest attractive face (or faces);

3. blend the target feature of the nearest attractive face (or faces) with the original face.

With high probability we obtain an aesthetic improvement. The effectiveness of these general steps depends on several factors, such as the dimensionality of the approach (2D or 3D) and the number of attractive face samples in the reference database.

We presented a first implementation of this idea in [26], focused on 2D face profiles and the target feature was the nose, which is one of the most distinctive face feature and object of many plastic surgery procedures. Also consider that beauty ratings of front and profile images are strongly correlated [19], and the nose shape dominates 2D profiles. During this work, we collected encouraging results supporting the effectiveness of the proposed approach (in Fig. 2, some results are shown). 

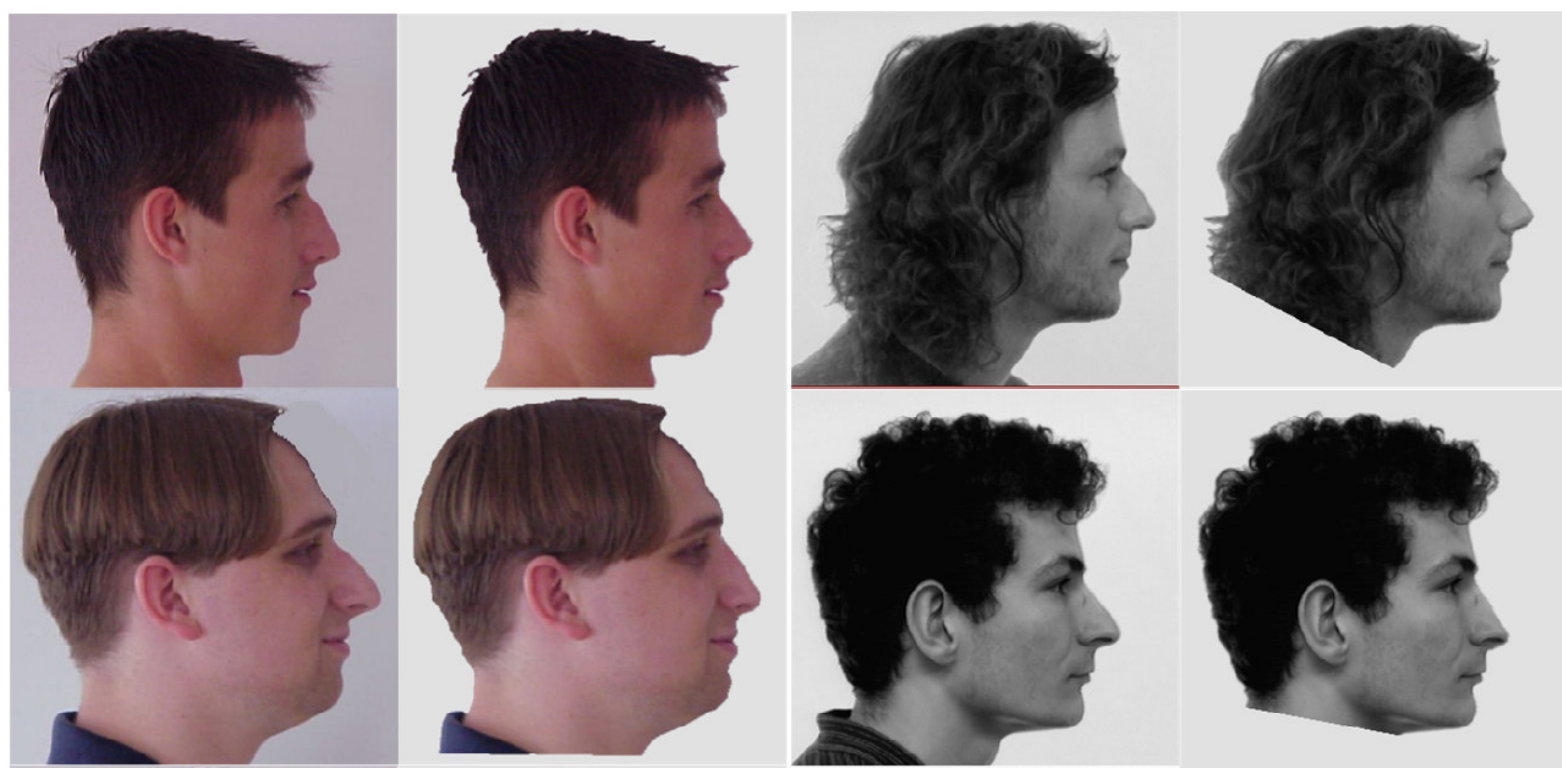

Fig.2. Aesthetic improvement for 2D profile: some results from our previous work [26].

Currently we are applying the presented approach to 3D face scans. It is clear that 3D shape conveys many more attractiveness elements than frontal or profile $2 \mathrm{D}$ images, and then allows constructing a much more effective plastic surgery support application. On the other side, working with 3D scans is more complex, computationally expensive and requires a 3D database of attractive faces. In the next section we illustrate the techniques used and the state of the project.

\section{Suggesting effective face surgery on the basis of 3D shapes}

\subsection{Constructing a 3D database of attractive faces}

The beautification process relies on a set of attractive or harmonious faces (the reference database). Unfortunately, not many 3D face databases exist, and they are essentially built to support face recognition research. In addition, to the authors' knowledge, no database rated for beauty exists. In any case, the available 3D databases, compared with 2D face databases, contain relatively few faces, and only a very small number of scans are useful for our purposes. According to preliminary estimates, a dense sampling of the manifold of beautiful faces in the face space could require several hundreds of samples.

In order to create a convenient reference DB, we are working in two directions. First, we are selecting the most attractive faces from the available 3D databases. Second, we launched an initiative (named "Faces for the Science") aimed at acquiring high-resolution 3D attractive face scans (Fig. 3). The initiative is backed by the Human Morphology Department of the University of Milan and involves professional modeling agencies in order to exploit specific professional expertise for the attractiveness rating process. The scanning campaign is in progress and so far we collected 49 three-dimensional face scans of both male and female multiethnic subjects, age between 16 to 30 years old. Data have been acquired with the VECTRA-CR-two-pod system [33], a portable non-contact passive stereoscopic scanner that guarantees full 180-degree capture. Each 3D model has approximately 90.000 vertices with an associated 10 Megapixel resolution texture. Initial scans are post processed to fill holes and remove spikes in their surface. Then, boundary artifacts, like hairs and dresses, are removed by trimming manually the faces (Fig. 4). We are currently investigating how to automate this process. 


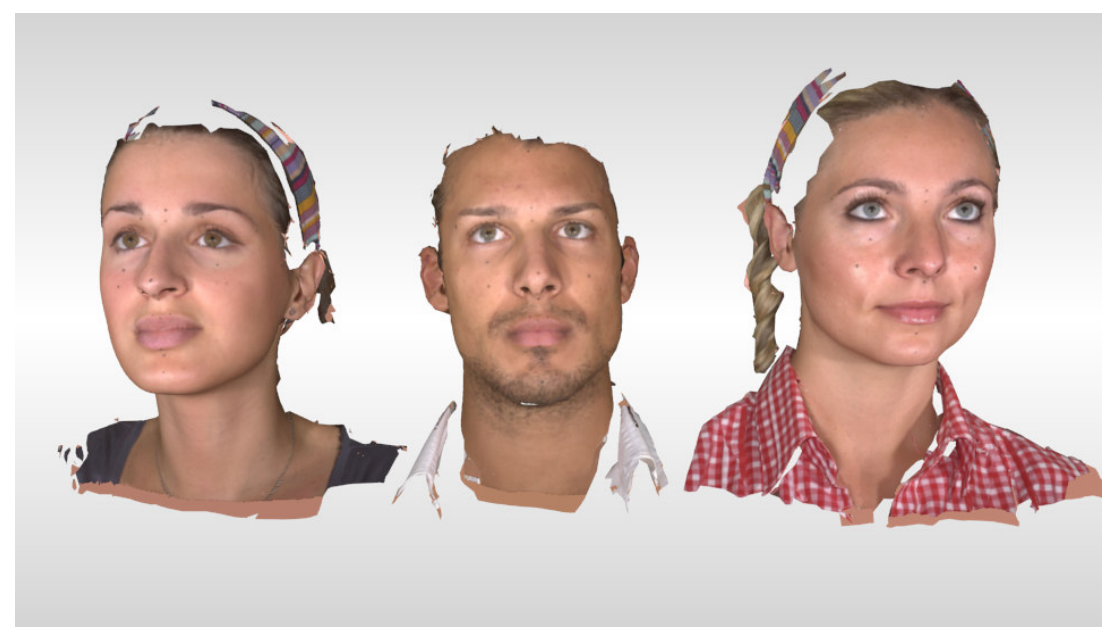

Fig. 3. 3D face scan database: raw data examples.

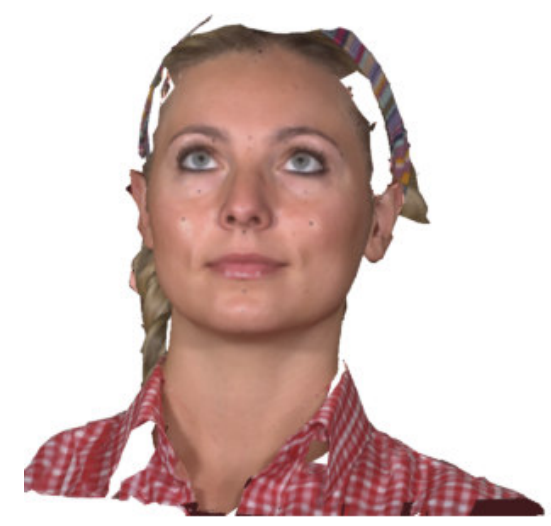

(a)

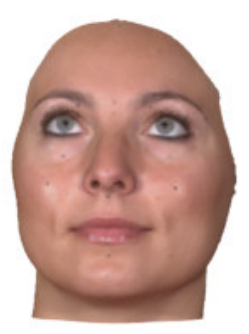

(b)

Fig. 4. A raw (a) and post-processed (b) scan.

Since samples in the reference database come from different sources, a normalization of the data is necessary. Differences in position, orientation and dimensions must be compensated. First, faces are aligned in 3D space by translating their centroid in the origin and making their symmetry axes, computed with the algorithm described in [27], coincident with the reference axes. In order to normalize dimensions, we compute the position of the nasion and subnasal repere points (Fig. 5) on the profile curve obtained slicing the mesh with the vertical symmetry plane and applying the method described in [26] (Fig. 6). Their distance gives an initial scaling factor. A finer alignment of the scans is finally performed using the Iterative Closest Point (ICP) algorithm [28] that registers two meshes performing rotation, translation and uniform scale transformations.

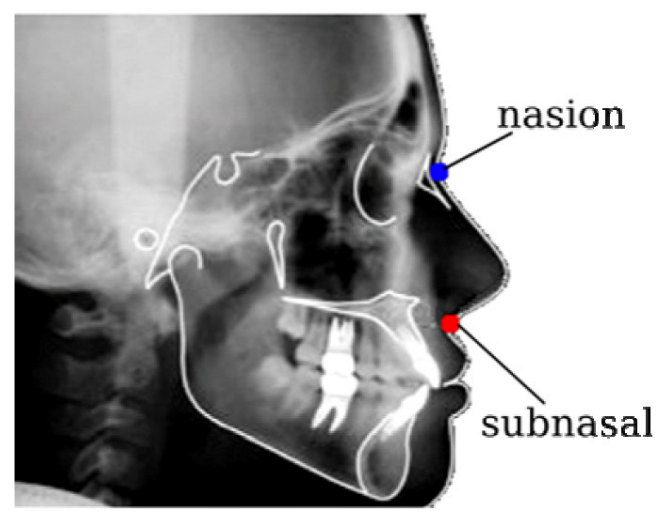

Fig. 5. Repere points: nasion and subnasal. 


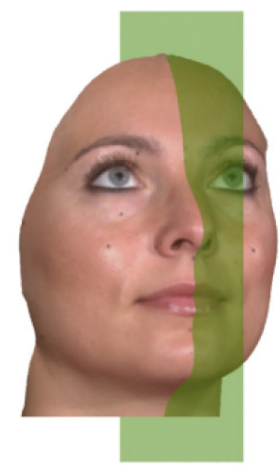

(a)

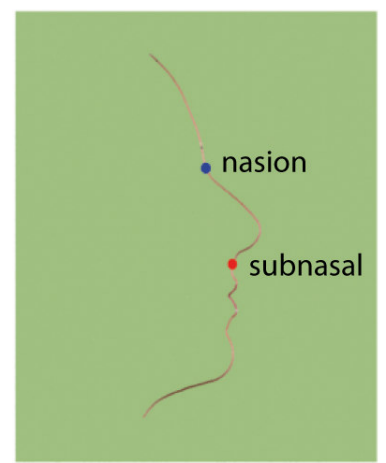

(b)

Fig. 6. Mesh (a) is sliced with the vertical symmetry plane to find the profile curve (b), where repere points are automatically identified.

In order to normalize the resolution, faces are re-sampled to obtain a point cloud on a uniform 2D $(h, \phi)$ grid (Fig. 7c) in the following way:

1. each mesh is sliced with an horizontal plane $(h)$ that is translated along the vertical axis by 512 steps between a minimum and a maximum value, obtaining 512 profile curves (Fig. 7a);

2. these curves are intersected with a vertical plane $(\phi)$ that is rotated from $0^{\circ}$ to $180^{\circ}$ around the vertical axis by 512 angular steps (Fig. $7 \mathrm{~b}$ ).

The final mesh is reconstructed applying a 3D Delaunay triangulation to the resulting point cloud (Fig. 7c).

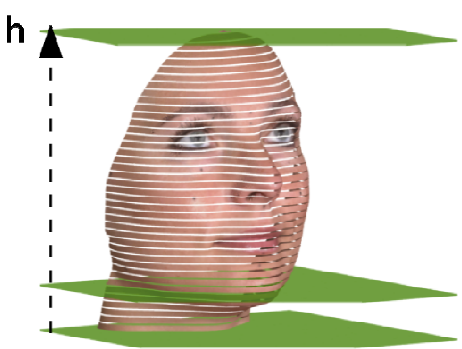

(a)

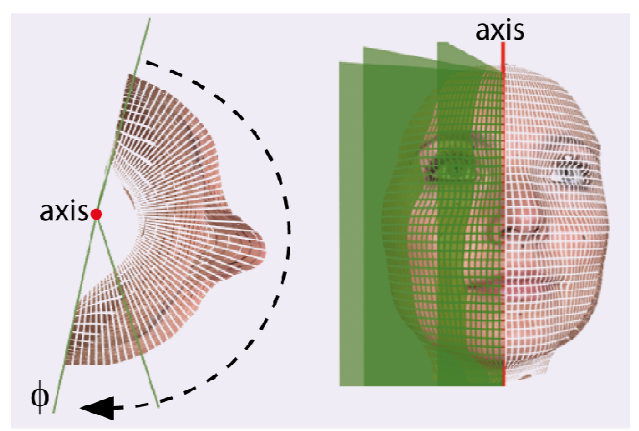

(b)

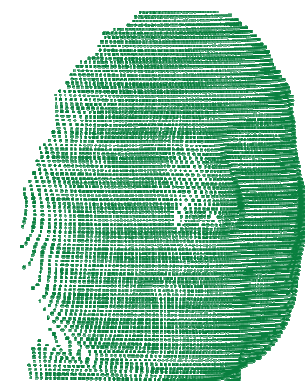

(c)

Fig. 7. Re-sampling process: (a) the mesh is sliced with a horizontal plane $h$ obtaining profile curves, (b) curves are intersected with a vertical plane $\phi$ that is rotated around the vertical axis. (c) Final point cloud.

\subsection{Implementing the 3D beautification approach}

Implementing the proposed approach to face beautification requires developing the following functions:

- selecting and removing a particular target feature (a part of the surface) on the patient's scan;

- removing the correspondent target feature from all faces present in the reference database;

- comparing the patient's scan with the faces of the database, all without the target feature

- merging the target feature of the most similar face (or faces) in the database with the patient's face

Patient's feature selection is performed interactively: the surgeon draws the contour of the feature he is interested in by picking points on the 3D surface (Fig. 8). 


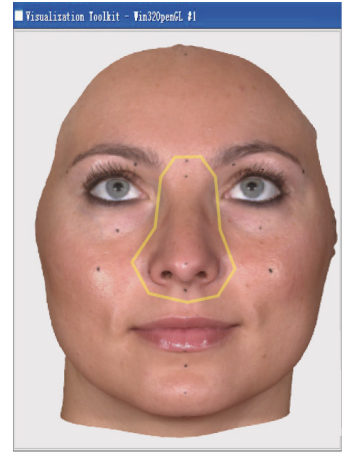

(a)

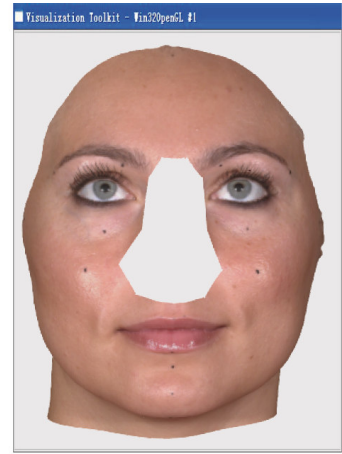

(b)

Fig. 8. Interactive feature selection: contour drawing (a); removal of the feature from the model (b).

Removing the same feature from the samples in the reference database requires identifying its contour on all of them. This, in turn, requires establishing first a dense point-to-point relationship between the input face and the reference faces. We approach the problem using the method proposed by Blanz and Vetter [29] for building their morphable 3D face model.

First, a 3D model $S$, re-sampled with the method described in the previous section, is transformed into a four-channel $512 \times 512$ 2D image $l(h, \phi)=\{\mathrm{R}(h, \phi), \mathrm{G}(h, \phi), \mathrm{B}(h, \phi), \mathrm{d}(h, \phi)\}$, where $(h, \phi)$ are the coordinates of a surface point, $\mathrm{R}(h, \phi), \mathrm{G}(h, \phi), \mathrm{B}(h, \phi)$ its texture values and $\mathrm{d}(h, \phi)$ its distance from the vertical axis (Fig. 9). Then, the correspondences between the points of two scans $S_{1}$ and $S_{2}$ can be computed applying a modified optical flow algorithm to their transformations $I_{1}$ and $I_{2}$. Currently, the Thirion's "daemon" registration algorithm $([30,31])$ is used for optical flow evaluation.

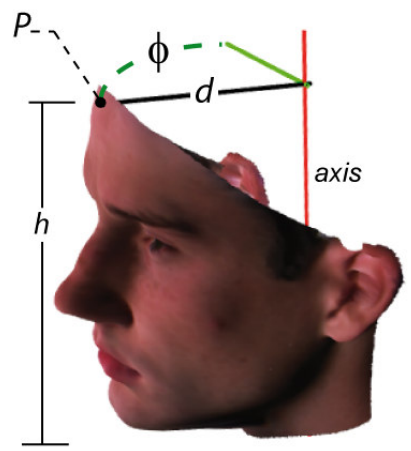

(a)

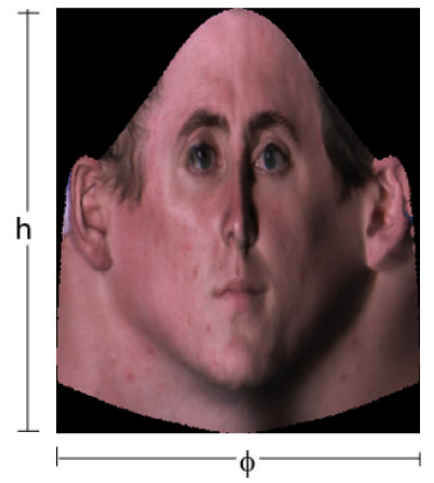

(b)

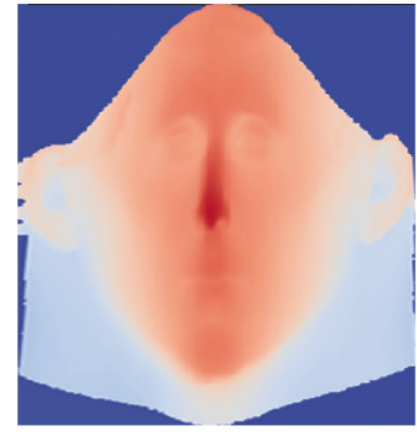

(c)

Fig. 9. The surface point $P$ corresponding to $(h, \varphi)$ coordinates (a). The $512 \times 512 R G B(b)$ and d channels of the $2 D$ transformation applied to the model shown in (a).

An example showing the feature selected in Fig. 8 and the corresponding contours on some faces of the reference DB is shown in Fig. 10.

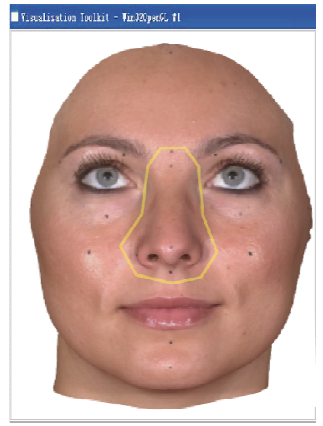

(a)

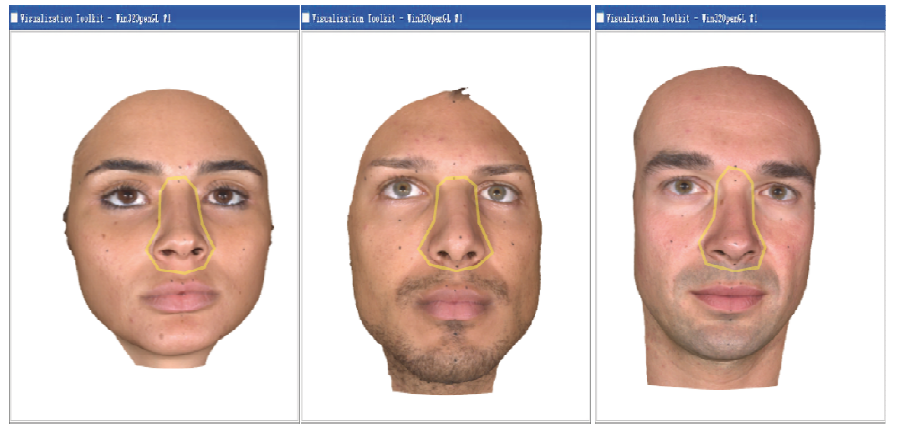

(b)

Fig. 10. Feature selection on the reference faces: the input selection (a) and the correspondent selection on reference $D B$ faces (b) 
Since evaluating the correspondences between each patient's scan and all the reference faces is computationally expensive, we use a faster technique. We introduce an average face $S_{0}$, and compute once for all the dense correspondences with the reference faces in the DB. Thus, given an input face we only compute its correspondences with the mean face, and the point-to-point relationships with each reference face can be quickly found by using the pre-computed data (Fig. 11).

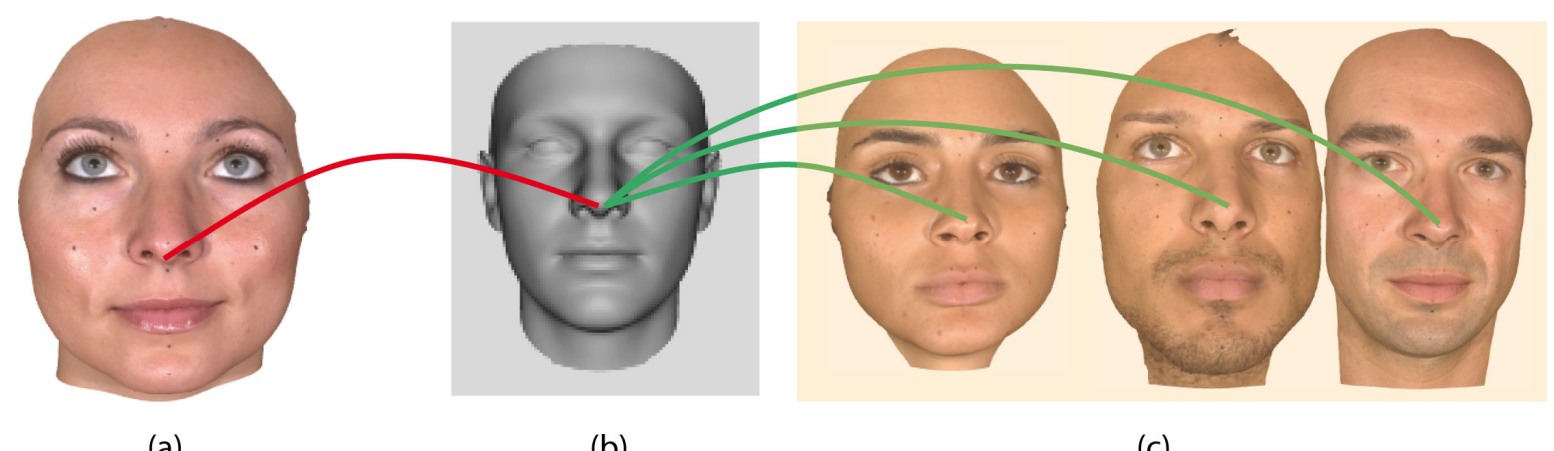

(a)

(b)

(c)

Fig. 11. A point of the input face (a) corresponds to a point on the average face (b) that, in turn, corresponds to a point on each face in the reference $D B(c)$.

Regarding face comparison, it is clear that a rigid comparison is not fit, since uniform scaling is immaterial from the point of view of attractiveness. The mean squared distance of correspondent points, minimized by the ICP algorithm, is used as measure of the distance between faces. We are investigating the opportunity to use an anisotropic ICP method where a not uniform scale transformation is involved in the various directions, since probably small differences in scale do not affect attractiveness. However, this point will be the object of further investigations.

Once the most similar face has been selected, we must paste its target feature onto the patient's face with a suitable blending. This requires: a) changing the scale of target feature to fit that of the receiving face; b) a seamless blending of the two surfaces. ICP algorithm already returns geometric transformation required to fit target feature and the receiving face. Two approaches are suitable for surface blending. The first one morphs the existent feature to stick onto the target feature (Fig. 12a). It is easy to implement by using a landmark-based algorithm available in the support library used in the implementation [32]. The second directly connects the target feature with the patient's face by knowing their point-to-point relationship (Fig. 13). A heuristic local deformation function smoothes unstitched geometry.

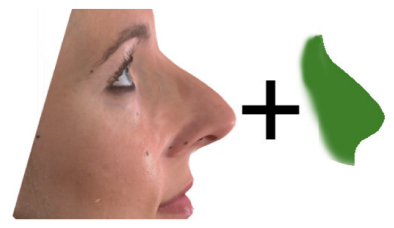

(a)

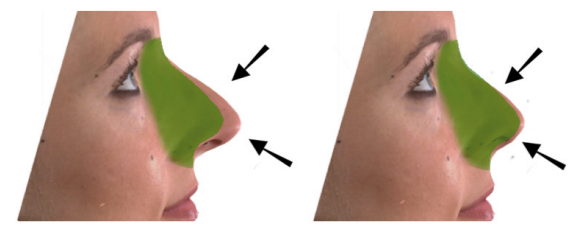

(b)

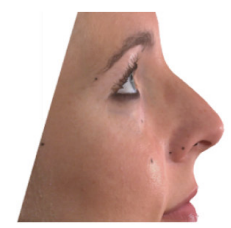

(c)

Fig. 12. The target feature (a, in green) is used to morph the existent feature (b) to obtain the final result (c).

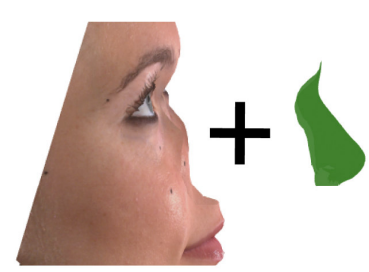

(a)

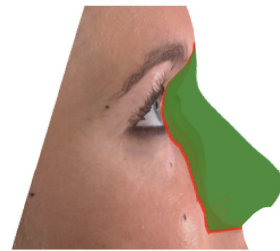

(b)

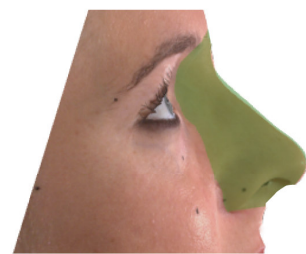

(c)

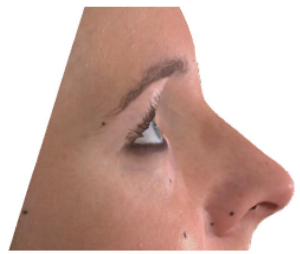

(d)

Fig. 13. The target feature ( $a$, in green) is connected to the patient's face (c) removing unstiched geometry (red area) and smoothing the joint area to obtain the final result (d).. 


\section{Conclusions}

This paper presents an application under development, aimed at supporting face surgery planning by automatically suggesting aesthetically effective shapes. The main idea relies on the prudent but safe assumption, supported by several recent studies, that there are many attractiveness prototypes, where the single features may be rather different but harmonize with the others. Then, suggesting aesthetically effective surgery can be done removing an unpleasant or disharmonic feature from the patient's face model and substitute it by the correspondent feature coming from the most similar face (or faces) present in a database of attractive faces. In a previous paper, we have presented this approach applied to 2D face profiles. Here we illustrate the full application of the general idea to $3 \mathrm{D}$ scans.

Due to the early stage of the tests, we are not yet able to report experimental results of this new tool. Several improvements are also under development. The optical flow algorithm for finding point-to-point correspondence could be improved. In particular we are testing different parameter values and different registration algorithms. Also comparison metric needs more investigation. The minimum average squared distance resulting from ICP appears effective, but, different distance measurement could be more fit to attractiveness. Finally, an effective practical tool requires a much wider database, encompassing several ethnicities and physiognomic types, and the two genders. More efforts will be devoted to grow the current reference database.

\section{Acknowledgements}

We want thanks Marie Biondini of the modeling agency "Indigo Fashion Toolbox" and Marco Todaro of the brand beauty fashion agency "Officina dello Stile", for their valuable support in the research and in the "Faces for the Science" initiative.

\section{References}

1. Bracaglia, R., Fortunato, R. and Gentileschi, S., (2005): "Secondary Rhinoplasty", Aesthetic Plastic Surgery, Vol.29, pp.230-239

2. Ozkul, T. and Ozkul, M.H., (2004): "Computer simulation tool for rhinoplasty planning", Comput. in Biol. and Med., Vol.34, pp.697-718

3. Rabi, S. and Aarabi, P., (2006): "Face Fusion: an automatic method for virtual plastic surgery", Proc. Int. Conf. on Information Fusion.

4. Gao, J., Zhou, M., Wang, H. and Zhang C., (2001): "Three dimensional surface warping for plastic surgery planning", IEEE proc.,pp.2016-2021.

5. Lee T., Lin, C. and Lin, H., (2001): "Computer aided prototype system for nose surgery", IEEE Trans. on Info. Tech. in Biomed., Vol.5, No.4, pp.271-278

6. Kavagiou, Z., Bello, F., Scott, G., Hamann, J. and Roberts, D., (2005): "Facial Plastic Surgery Planning Using a 3D Surface Deformation Tool", Medicine Meets Virtual Reality 13: The Magical Next Becomes the Medical Now, Amsterdam, IOS Press, pp.247-250.

7. Bashour, M., (2006): "History and Current Concepts in the Analysis of Facial Attractiveness", Plastic and Reconstructive Surgery, Vol.118, No.3, pp.741-756.

8. Cunningham, M.R., Roberts, A.R., Barbee, A.P., Druen, P.B., (1995): "Their ideas of beauty are, on the whole, the same as ours: Consistency and variability in the crosscultural perceptions of female attractiveness", Journal of Personality and Social Psycology, Vol.68, pp.261-279.

9. Etcoff, N., (1994): "Beauty and the beholder", Nature, Vol.368, pp.186-187.

10. Jones, D., (1996): "Physical Attractiveness and the Theory of Sexual Selection: Results from Five Populations", University of Michigan Press, Ann Harbour.

11. Zhao, W., Chellappa, R., Philips, P.J., and Rosenfeld, A, (2003): "Face Recognition, a literature survey", ACM Computing Surveys, Vol.35, No.4, pp.399-458

12. Edler, R., Agarwal P., Wertheim, D. and Greenhill, D., (2006): "The use of anthropometric proportion indices in the measurement of facial attractiveness", European Journal of Orthodontics, Vol.28, pp.274-281.

13. Farkas, L.G., Hreczko, T.A., Kolar, J.C., Munro, I.R., (1985): "Vertical and horizontal proportions of the face in young adult North American Caucasians: Revision of neoclassic canons", Plastic \& Reconstructive Surgery, Vol.75, pp.328-337. 
14. Aarabi, P., Hughes, D., Mohajer, K., Emami, M., (2001): "The automatic measurement of facial beauty", IEEE Proc. Int. Conf. on Systems, Man and Cyb.

15. Bashour, M., (2006): "An objective system for measuring facial attractiveness", Plastic \& Reconstructive Surgery, Vol.118, pp.757-774.

16. Eisenthal, Y., Dror, G. and Ruppin, E., (2006): "Facial attractiveness: beauty and the machine", Neural Computation, Vol.18, pp.119-142.

17. Gunes, H., Piccardi, M. and Jan, T., (2004): "Comparative beauty classification for pre-surgery planning", IEEE Proc. Int. Conf. on Systems, Man and Cybernetics, pp.2168-2174.

18. Moss, J., Linney, A. and Lowey, M., (1995): "The use of three-dimensional techniques in facial Esthetics", Seminar in Orthodontics, Vol.1, pp.94-104.

19. Valenzano, D., Mennucci, A., Tartarelli G. and Celerino, A., (2006): "Shape analysis of female face attractiveness", Vision Research, Vol.46, pp.1282-1291.

20. Leyvand, T., Cohen-Or, D., Dror G. and Lishinski, D., (2006): "Digital face beautification", ACM Siggrapph 2006, Technical Sketch T.

21. Alley, T.R. and Cunningham, M.R., (1991): "Average faces are attractive, but very attractive faces are not average", Psychological science, Vol.2, pp.123-125.

22. Perret, D., May, K. and Yoshikawa, S., (1994): "Facial shape and judgements of female attractiveness", Nature, Vol.368, pp.239-242.

23. Le, T., Farkas, L., Ngim, R., Levin, L. and Forrest, C., (2002): "Proportionality in asian and north American Caucasian faces using neoclassic facial canons as criteria", Aesthetic Plastic Surgery, Vol.26, pp.64-69.

24. Rosch, E., (1978): "Principles of categorization”, In Rosch, E. and Lloyd B., (1978): "Eds. Cognition and Categorization", Erlbaum, 1978.

25. Lee, T., Sun, Y., Lin, Y., Lin, L. and Lee, C., (1999): "Three-dimensional facial model reconstruction and plastic surgery simulation", IEEE Trans. on Info. Tech. in Biomed., Vol.3, No.3, pp.214-220.

26. Bottino, A., Laurentini, A., Rosano, L., (2008): "A New Computer-aided Technique for Planning the Aesthetic Outcome of Plastic Surgery", Proceedings of 16th Int. Conf. on Computer Graphic, Visualization and Computer Vision WSCG 2008, PIzen (Cz)

27. Pan, G., Wang, Y., Qi, Y. and Wu, Z., (2006): "Finding Symmetry Plane of 3D Face Shape", Proceedings of the 18th International Conference on Pattern Recognition, vol. 3, pp.1143-1146.

28. Zhang, Z., (1994): "Iterative point matching for registration of free-form curves and surfaces", Int. J. Comput. Vision, Vol. 13, N0. 2, pp.119--152

29. Blanz, V. and Vetter, T., (2003): "Face Recognition Based on Fitting a 3D Morphable Model", IEEE Trans. Pattern Anal. Mach. Intell., vol. 25, no. 9, pp. 1063-1074.

30. Thirion, J. P., (1995): "Fast non-rigid matching of 3D medical image." Technical report, Research Report RR-2547, Epidure Project, INRIA Sophia.

31. Thirion, J.P., (1998): "Image matching as a diffusion process: an analogy with maxwell's demons." Medical Image Analysis, vol. 2, no.3, pp.243-260.

32. VTK visualization toolkit software system (accessed 2010): http://www.vtk.org

33. Canfield Imaging System (accessed 2010): http://www.canfieldsci.com 